\title{
Lung transplantation for COVID-19-associated acute respiratory distress syndrome after extended use of extra corporeal membrane oxygenation: a case report
}

\author{
Soomin Yang ${ }^{1}$, Sung Yoon Lim¹, Myung Jin Song ${ }^{1}$, Jaewon Beom², Young-Jae Cho ${ }^{1}$ \\ ${ }^{1}$ Department of Internal Medicine, Seoul National University Bundang Hospital, Seongnam, Korea \\ ${ }^{2}$ Department of Rehabilitation Medicine, Seoul National University Bundang Hospital, Seongnam, Korea
}

Background: Although lung can recover using extracorporeal membrane oxygenation (ECMO) support in patients with severe COVID-19-associated acute respiratory distress syndrome (ARDS), lung transplantation is lifesaving for those who develop irreversible lung injury.

Case report: We report a patient with COVID-19 ARDS who underwent successful lung transplantation after 97 days of bridging with ECMO support. A previously healthy 71-year-old male with no history of underlying diseases was diagnosed with COVID-19. He was intubated for severe ARDS; however, positive pressure ventilation with prone positioning and corticosteroid treatment failed to improve his hypoxemia. For refractory hypoxemia, venovenous ECMO was initiated via bi-femoral configuration performed at the bedside. When the patient was negative for SARS-CoV-2 during the third week of ECMO, the patient was transferred to the general intensive care unit (ICU), and we implemented active ICU rehabilitation. We kept the patient awake, and he was communicating frequently with his family using a video call. Despite the improvement of hypoxemia after ECMO support, hypercapnia persists even with sweep gas greater than $5 \mathrm{~L} / \mathrm{min}$. We changed the membrane oxygenator twice and then pumpless interventional lung assist was implanted percutaneously for severe hypercapnia. Double lung transplantation was performed on the day 98th of ECMO. No bleeding or primary graft dysfunction was observed within the first 72 hours. The patient was liberated from mechanical ventilation on postoperative day 5 . Currently, the patient can undergo active physical rehabilitation.

Conclusions: This case suggests that it is feasible to wait for the native lung recovery or lung transplant with extended use of ECMO support in patients with COVID-19-associated ARDS. While waiting, concurrent active rehabilitation with ECMO might improve outcomes after lung transplantation.

Corresponding author: Sung Yoon Lim

E-mail: nucleon727@gmail.com

\footnotetext{
(c) The Korean Society for Transplantation

This is an Open Access article distributed under the terms of the Creative Commons Attribution Non-Commercial License (http://creativecommons.org/licenses/by-nc/4.0/) which permits unrestricted non-commercial use, distribution, and reproduction in any medium, provided the original work is properly cited.
} 